\title{
STUDY ON THE EXTRACTION PROCESS OF TOTAL ANTHRAQUINONES IN RADIX ET RHIZOMA RHEI AND THEIR ANTILIPEMIC EFFECTS
}

\section{Hui-chun Xie*, Jun Shang}

The key laboratory of education ministry on environments and resources in Tibetan Plateau, Qinghai-Normal University, Xining 810008, China

*E-Mail: bpinweog@yeah.net

\begin{abstract}
Background: Radix et Rhizoma Rhei has a gastric mucosal protective effect, major anti-gastritis and anti-peptic ulcer active constituents were emodin, aloe-emodin, chrysophanol, rhein, etc. The objective of the study was the extraction process of total anthraquinones in Radix et Rhizoma rhei and their antilipemic effects.

Materials and Methods: Orthogonal experiment, UV spectrophotometry and conventional antilipemic effect determination method were used to optimize the extraction process, and to determine the total anthraquinone content, as well as blood levels of total cholesterol, triglycerides, HDL and LDL.

Results: Ethanol concentration, extraction time and ethanol amount had significant influences on the extraction of total Radix et Rhizoma rhei anthraquinones, total Radix et Rhizoma rhei anthraquinones could significantly reduce blood levels of total cholesterol, triglycerides, HDL and LDL.

Conclusion: The optimum extraction process was two times extraction of Radix et Rhizoma rhei with 70\% ethanol, the amounts of solvent of 8 folds and 5 folds, successively, and the extraction time of $60 \mathrm{~min}$ each. In addition, this extract has an antilipemic effect in mice.
\end{abstract}

Keywords: Radix et Rhizoma Rhei; total anthraquinones; orthogonal test; anti-lipids

\section{Introduction}

Radix et Rhizoma rhei is the dried root and rhizome of Rheum palmatum L., R. tanguticum Maxim. ex Balf. or R. officinale Baill. of the family Polygonaceae, whose major active constituent was anthraquinone derivatives (Li et al., 2006). Radix et Rhizoma Rhei has a gastric mucosal protective effect, major anti-gastritis and anti-peptic ulcer active constituents were emodin, aloe-emodin, chrysophanol, rhein, etc. (Ding et al., 2007; Wang et al., 1997; Zhang, 2008). In recent years, macroporous resin is widely used in the field of purification and refinement of TCM, which is characterized by large adsorption capacity, simple regeneration and reliable method, macroporous resin is especially suitable for purification and isolation of large acute chemical constituents, while achieving a good isolation effect in the refinement of anthraquinones, flavonoids, saponins, alkaloids and other active constituents of TCM, macroporous resin can also significantly reduce the yield of solid matter, and further reduce the dose (Li, 2007; Chen et al., 2009). This study will focus on the investigation of extraction process of total anthraquinones in Radix et Rhizoma Rhei and their antilipemic effects.

\section{Materials and Methods}

Instruments and reagents

751G UV spectrophotometer (Shanghai Analytical Instrument Factory); electronic analytical balance (Shanghai Analytical Instrument Factory). All reagents were of analytical grade; and water was distilled water.

Drugs

Radix et Rhizoma Rhei was purchased from Sichuan TCM Decoction Pieces Co., Ltd., which was identified by Professor Lu Feng from the Department of Pharmacognosy of our institute to be consistent with relevant requirements under subparagraph Radix et Rhizoma rhei in the "Chinese Pharmacopoeia" 2010 edition Vol. 1. Reference substance emodin (99.8\%) was purchased from the National Institute for the Control of 
http://dx.doi.org/10.4314/ajtcam.v11i2.22

Pharmaceutical and Biological Products, batch number: G2365-6395.

Animals

24 healthy adult Kunming mice from the SPF center of Qinghai-Normal University, half male and half female, weighing 20 25 g, were randomly divided into three groups. All experimental procedures were approved by the Animal Research Committee.Ethics

\section{Methods and Results}

Plotting of standard curves and establishment of content determination method

Emodin reference solution: $4.9 \mathrm{mg}$ of emodin reference substance was accurately weighed, dissolved in an appropriate amount of methanol, diluted to the mark in a $50 \mathrm{~mL}$ volumetric flask, and shaken well, which was used as the reference solution. Plotting of standard curves: $0.5,1,1.5,2,2.5,3$ and $3.5 \mathrm{ml}$ of emodin reference solutions $(0.098 \mathrm{mg} / \mathrm{ml})$ were accurately drawn, placed in $25 \mathrm{ml}$ flasks, and stained by addition of $10 \mathrm{ml}$ of $0.5 \%$ magnesium acetate methanol solution, respectively. Then their volumes were diluted to $25 \mathrm{ml}$ with methanol, and shaken well. Absorbance was measured at $510 \mathrm{~nm}$ with $0.5 \%$ magnesium acetate methanol solution as the blank control. Regression equation was calculated as $\mathrm{A}=3714 \mathrm{C}+0.00765 \mathrm{R}=0.9990$

\section{Preparation of test solution}

$20 \mathrm{ml}$ of extract was accurately drawn, placed in a $250 \mathrm{ml}$ round bottom flask, added with $50 \mathrm{ml}$ of $2 \% \mathrm{H}_{2} \mathrm{SO}_{4}$ and $70 \mathrm{ml}$ of chloroform, and heated and hydrolyzed in a water bath for $2 \mathrm{~h}$, after cooling, chloroform layer was separated and taken, and the hydrolysate was again extracted three times with chloroform, until the color of chloroform layer turned light. The chloroform solutions were combined, adjusted to neutral $\mathrm{pH}$, chloroform was removed, and the residue was dissolved in methanol, and diluted to the mark in a $25 \mathrm{ml}$ volumetric flask, which was used in content determination.

\section{Water extraction method}

$10 \mathrm{~g}$ of Radix et Rhizoma Rhei was accurately weighed, and extracted by conventional reflux extraction, each extract was concentrated, respectively, and diluted to the mark in $50 \mathrm{ml}$ volumetric flasks, after treatment in accordance with the method in the "Preparation of test solution", $1 \mathrm{ml}$ was precisely pipetted into a $50 \mathrm{ml}$ volumetric flask, stained, and content was determined and calculated according to the standard curve plotting method in the "Plotting of standard curves and establishment of content determination method" (Table. 1).

Table 1: Total anthraquinone yield by water extraction of Radix et Rhizoma Rhei

\begin{tabular}{cccc}
\hline Number of times & Water volume $(\mathrm{ml})$ & Time $(\mathrm{min})$ & Total anthraquinone yield $(\%)$ \\
\hline 1 & 80 & 60 & 0.845 \\
2 & 60 & 30 & 0.526 \\
3 & 40 & 30 & 0.265 \\
4 & 40 & 30 & 0.254 \\
5 & 40 & 30 & 0.189 \\
\hline
\end{tabular}

Ethanol extraction method

$10 \mathrm{~g}$ of Radix et Rhizoma Rhei was accurately weighed, and extracted under reflux with $70 \%$ ethanol, treatment and content determination methods were the same as in the "Water extraction method"Tables. 2, 3).

Table 2: Total anthraquinone yield by ethanol extraction of Radix et Rhizoma Rhei

\begin{tabular}{cccc}
\hline Number of times & Solvent volume $(\mathrm{ml})$ & Time $(\mathrm{min})$ & Total anthraquinone yield (\%) \\
\hline 1 & 50 & 60 & 2.025 \\
2 & 40 & 30 & 0.512 \\
3 & 30 & 30 & 0.348 \\
4 & 30 & 30 & 0.169 \\
\hline
\end{tabular}


Xie and Shang Afr J Tradit Complement Altern Med. (2014) 11(2):358-362

http://dx.doi.org/10.4314/ajtcam.v11i2.22

Table 3: Results of total anthraquinone yield by different extraction methods of Radix et Rhizoma Rhei

\begin{tabular}{ccc}
\hline Extraction method & Total anthraquinone yield (\%) & Extraction rate (\%) \\
\hline Reference medicinal material & 5.282 & - \\
Ethanol extraction method & 3.054 & 57.819 \\
Water extraction method & 2.079 & 39.360 \\
\hline
\end{tabular}

\section{Optimization of ethanol extraction process conditions}

Investigated factors and their levels

Four factors, namely ethanol concentration, extraction time, ethanol amount and extraction times, were selected, and each factor had three levels; the level of factor table was drawn as shown in Table 4.

Table 4: Levels and factors of ethanol extraction process of total anthraquinones in Radix et Rhizoma Rhei

\begin{tabular}{ccccc}
\hline Level & \multicolumn{3}{c}{ Factor } \\
\hline & A Ethanol concentration (\%) & B Ethanol amount (folds) & C Extraction time (min) & D Extraction times (times) \\
1 & $50 \%$ & $4,3,3$ & $60,60,45$ & 1 \\
2 & $70 \%$ & $6,4,3$ & $60,45,30$ & 2 \\
3 & $90 \%$ & $8,5,4$ & $45,30,30$ & 3 \\
\hline
\end{tabular}

Note: if $\mathrm{D}$ is 1 time, it corresponds to the first digit values of $\mathrm{B}, \mathrm{C}$; if $\mathrm{D}$ is 2 times, it corresponds to the second digit values of $\mathrm{B}$, C; and if $\mathrm{D}$ is 3 times, it corresponds to all values of $\mathrm{B}, \mathrm{C}$.

\section{Optimization experiment of ethanol extraction process conditions and results}

$10 \mathrm{~g}$ of Radix et Rhizoma Rhei medicinal material was separately weighed, extracted, concentrated and then diluted to the mark in $50 \mathrm{ml}$ volumetric flasks according to the $\mathrm{L}_{9}\left(3^{4}\right)$ orthogonal design program, test solution was prepared under the method prescribed in "Preparation of test solution", and total anthraquinone content was determined according to the method in "Ethanol extraction method". Experimental program and the results are shown in Table 5.

Range analysis results in Tab. 5 show that the factors A, B, C and D had different degrees of influence on extraction of total anthraquinones in Radix et Rhizoma Rhei, which were $A>C>B>D$, the combination of optimum extraction process was: three times of extraction with 70\% ethanol, solvent amount of 8, 5 and 4 folds and extraction time of 45, 30 and 30 min, respectively. ANOVA was performed with total anthraquinone yield as an index. The results are shown in Table 6.

ANOVA results showed that factor A had a significant influence on the extraction of total anthraquinones in Radix et Rhizoma Rhei, after comprehensive consideration of various factors and actual production, the optimum extraction process of total anthraquinones in Radix et Rhizoma Rhei was determined as $\mathrm{A}_{2} \mathrm{~B}_{2} \mathrm{C}_{1} \mathrm{D}_{2}$, i.e. two times extraction with $70 \%$ ethanol, a 8-fold amount of solvent in the first extraction, and a 5 -fold amount of solvent in the second extraction, as well as extraction time of 60 min each. Scale-up experiment on the determined process conditions showed that the yield was basically stable.

Antilipemic effect of total anthraquinones in Radix et Rhizoma Rhei (Luo et al., 2006; Jin et al., 2006; Dong, 2006)

\section{Administration method}

Mice were administered i.g. with $3.0 \mathrm{~g} / \mathrm{kg}, 5.0 \mathrm{~g} / \mathrm{kg}$ and $10.0 \mathrm{~g} / \mathrm{kg}$ of total Radix et Rhizoma Rhei anthraquinone extracts or an equivalent volume of saline daily for a total of $10 \mathrm{~d}, 1 \mathrm{~h}$ after the last administration, blood samples were collected to determine blood levels of total cholesterol, triglycerides, HDL and LDL.

\section{Determination method}

Total cholesterol and triglyceride levels were determined by enzymatic colorimetry; HDL and LDL levels were determined by clearance 
Xie and Shang Afr J Tradit Complement Altern Med. (2014) 11(2):358-362

http://dx.doi.org/10.4314/ajtcam.v11i2.22

method. The results are shown in Tables 7 and 8.

Table 5: Orthogonal experimental program and results

\begin{tabular}{ccccccc}
\multicolumn{6}{c}{ Table 5: Orthogonal experimental program and results } \\
\cline { 2 - 6 } Test No. & A & B & C & D & Total anthraquinone yield (\%) \\
\hline 1 & 1 & 1 & 1 & 1 & 0.875 \\
2 & 1 & 2 & 2 & 2 & 0.876 \\
3 & 1 & 3 & 3 & 3 & 0.901 \\
4 & 2 & 1 & 2 & 3 & 1.402 \\
5 & 2 & 2 & 3 & 1 & 1.180 \\
6 & 2 & 3 & 1 & 2 & 1.670 \\
7 & 3 & 1 & 3 & 2 & 1.063 \\
8 & 3 & 2 & 1 & 3 & 1.312 \\
9 & 3 & 3 & 2 & 1 & 1.315 \\
I & 2.632 & 3.339 & 3.879 & 3.356 & \\
II & 4.230 & 3.354 & 3.569 & 3.589 & \\
III & 3.679 & 3.912 & 3.126 & 3.721 & \\
R & 1.632 & 0.543 & 0.756 & 0.256 & \\
\hline
\end{tabular}

\begin{tabular}{llllll}
\multicolumn{7}{c}{ Table 6: ANOVA table } \\
\cline { 2 - 6 } Source of variation & Sum of Squares & Degrees of Freedom & Variance & F value & Significance \\
\hline A & 0.456 & 2 & 0.230 & 38.18 \\
B & 0.065 & 2 & 0.035 & 5.66 \\
C & 0.090 & 2 & 0.044 & 7.49 \\
Error & 0.012 & 2 & 0.006 & \\
\hline
\end{tabular}

$\mathrm{F}_{0.05}(2,2)=19.00, \mathrm{~F}_{0.01}(2,2)=99.00$

Table 7: Effects of total Radix et Rhizoma Rhei anthraquinones on serum levels of total cholesterol and triglycerides

\begin{tabular}{ccc}
\hline Group & Total cholesterol & Triglycerides \\
\hline Normal saline & $2.831 \pm 0.18$ & $1.468 \pm 0.10$ \\
Small dose & $2.401 \pm 0.16 *$ & $1.203 \pm 0.13 *$ \\
Medium dose & $2.212 \pm 0.15 * *$ & $1.152 \pm 0.08 * *$ \\
Large dose & $2.020 \pm 0.14 * *$ & $1.023 \pm 0.09 * *$ \\
\hline
\end{tabular}

Comparison with the normal saline group, $* \mathrm{P}<0.05 ; * * \mathrm{P}<0.01 ; \mathrm{n}=8$

As can be seen from Tab. 7, total Radix et Rhizoma Rhei anthraquinones could significantly reduce serum total cholesterol and triglyceride levels, and their effects exhibited apparent dose-dependence. As can be seen from Tab. 8, total Radix et Rhizoma Rhei anthraquinones could significantly reduce serum HDL and LDL levels, and their effects exhibited apparent dose-dependence.

Table 8: Effects of total Radix et Rhizoma Rhei anthraquinones on serum levels of HDL and LDL ( \pm \pm s) $\mathrm{mmol} / \mathrm{L}$

Comparison with the normal saline group, $* \mathrm{P}<0.05 ; * * \mathrm{P}<0.01 ; \mathrm{n}=8$

\begin{tabular}{ccc}
\hline Group & HDL & LDL \\
\hline Normal saline & $1.02 \pm 0.08$ & $0.46 \pm 0.02$ \\
Small dose & $1.18 \pm 0.10 * *$ & $0.35 \pm 0.03 *$ \\
Medium dose & $1.29 \pm 0.11 * *$ & $0.30 \pm 0.02 * *$ \\
Large dose & $1.50 \pm 0.11 * *$ & $0.31 \pm 0.03 * *$ \\
\hline
\end{tabular}

\section{Discussion}

Radix et Rhizoma Rhei was extracted with water and ethanol, respectively, and total anthraquinone content was measured, which was higher in the latter, indicating that ethanol was a more ideal extracting solvent. Content determination was performed according to the method 
http://dx.doi.org/10.4314/ajtcam.v11i2.22

prescribed in the Chinese Pharmacopoeia, and total anthraquinone content in medicinal Radix et Rhizoma Rhei was determined to be 5.282\%. After comparison between water and ethanol extraction processes, ethanol extraction process was determined to be more desirable. Analysis of orthogonal experimental results showed that the ethanol concentration had a significant influence on extraction of total Radix et Rhizoma Rhei anthraquinones, after comprehensive consideration of various factors, and combined with actual production, the optimum extraction process of total anthraquinones in Radix et Rhizoma Rhei was determined as two times extraction with 70\% ethanol, solvent amount of 8 and 5 folds, successively, and extraction time of 60 min each.

The present study found that total Radix et Rhizoma Rhei anthraquinones could reduce serum total cholesterol level, increase HDL level, lower LDL and VLDL levels, reduce LDL and prevent excessive LDL oxidation in mice. Total Radix et Rhizoma Rhei anthraquinones reduce fat deposition in the coronary artery wall through the above actions, thereby reducing the incidence of atherosclerosis. The results showed that total reducing the incidence of anthraquinones can markedly reduce blood lipid level, lower serum total cholesterol level, increase HDL level and lower LDL and VLDL levels in mice. Total reducing the incidence of anthraquinones have a significant hypolipidemic effect.

\section{Acknowledgements}

This work was supported by the hypolipidemic material base of Rhubard 2013-z-053.

\section{References}

1. Chen, Q. (2009). Application of macroporous adsorption resin in the research of new TCM preparation process. Journal of Fujian University of Traditional Chinese Medicine, 19(2): 67-70.

2. Ding, Y., and Huang, Z.H. (2007). Research progress in pharmacological effects of emodin. Pharmacology and Clinics of Chinese Materia Medica, 23(5): 236-238.

3. Dong, J.M. (2006). Study on the blood lipid regulating and anti-atherosclerotic effects of GLA powder in animals. Clinical Journal of Traditional Chinese Medicine, 18(2): 135-137.

4. Jin, S.J., Jiang, Y.X., Hu, X.Z., and Liu, J. (2006). Effects of Tangweikang on blood glucose, serum insulin and lipids in experimental insulin resistance rats. West China Journal of Pharmaceutical Sciences, 21(2): 126-129.

5. Li, C. (2007). Application progress of macroporous resin in isolation of Chinese medicinal constituents. Hubei Journal of Traditional Chinese Medicine, 29(10): 64-64.

6. Li, M., Li, L.X., Liu. Y., and Liu, Y. (2006). Study survey on Rhubarb in Recent years. World Science and Technology---Modernization of Traditional Chinese Medicine, 8(4): 34-40.

7. Luo, C.B., and Lan, J.Q. (2006). Blood lipid regulating effect of Qingzhiling in hyperlipidemic model rats. Journal of Yichun University, 28(2): 96-98.

8. Wang, H.H and Chung, J.G. (1997). Emodin-induced inhibition of growth and DNA damage in the Helicobacter pylori. Current Microbiology, 35(5): $262-266$.

9. Zhang, R.X. (2008). Brief investigation on effects of rhubarb other than purgative effect. Chinese Medicine Modern Distance Education of China, 6(10): 1217-1218. 